Artículo original

\title{
EFECTO DEL PROGRAMA DE ALIMENTACIÓN Y NUTRICIÓN EN LA RECUPERACIÓN DE PERSONAS CON POBREZA EXTREMA AFECTADAS DE TUBERCULOSIS; PROVINCIA DE TACNA, 2013.
}

Effect of the food and nutrition program on the recovery of people with extreme poverty affected by tuberculosis; Province of Tacna, 2013.

\author{
Victoria Calderón Fernández ${ }^{1}$
}

\section{RESUMEN}

El propósito fue conocer el efecto del Programa de Alimentación y Nutrición en la recuperación de personas con pobreza extrema afectadas de tuberculosis en la Provincia de Tacna durante el año 2013. Estudio explicativo de corte transversal, se revisaron 467 historias clínicas de pacientes que ingresaron a tratamiento por tuberculosis, encontrando 33 personas con pobreza extrema afectadas de tuberculosis, obteniendo información de su condición social, enfermedad, y a quienes se entrevista acerca del patrón de consumo de alimentos de la canasta de víveres del PANTBC.Para el análisis se tomó en cuenta las características socioeconómicas, evolución de la enfermedad, remisión de la sintomatología, evaluación nutricional según el Índice de Masa Corporal (IMC) al inicio y final del tratamiento. Todos los pacientes ingresaron al Programa con Diagnóstico de tuberculosis sensible y al término del tratamiento su condición fue de curado. En el $90.9 \%$ remitió la sintomatología, con valoración clínica y bacteriológica favorable. Del total de pacientes que ingresaron con bajo peso el $70 \%$ culminó con peso normal.

Palabras clave: tuberculosis, pobreza extrema, recuperación, nutrición.

\section{ABSTRACT \\ Objective. Determine the effect of Food and Nutrition Program in the recovery of people affected extreme poverty of tuberculosis in the province of Tacna in 2013. Design explanatory cross-sectional study. Materials and Methods: 467 medical records of patients admitted a treatment for tuberculosis were reviewed, finding 33 people in poorness extreme, obtaining information from their social condition, disease, and who are interviewed about the pattern of consumption food basket of PANTBC. For the analysis took into account the socio-economic characteristics, disease progression, remission of symptoms, nutritional assessment according to the Body Mass Index (BMI) at the beginning and end of treatment. Results: All patients entered the program with sensitive tuberculosis diagnosis and treatment at the end of his condition was cured. In $90.9 \%$ he referred}

the symptoms, with favorable clinical and bacteriological assessment. Of the total patients admitted underweight $70 \%$ culminated with normal weight.

Keywords: tuberculosis, extreme poverty, recovery, nutrition.

\section{INTRODUCCIÓN}

La tuberculosis en Tacna sigue siendo un problema de Salud Pública; siendo la cuarta Región en el año 2013 de mayor incidencia de tuberculosis en el Perú, después de Lima, Callao y Madre de Dios. En Tacna la atención integral de las personas con tuberculosis asegura la administración de medicamentos en forma gratuita en los Establecimientos de Salud del Ministerio de Salud y la entrega de una canasta de víveres mensual a cargo de la Municipalidad Provincial de Tacna por el Programa de Alimentación y Nutrición para paciente ambulatorio con tuberculosis y familia (PAN TBC). Según el informe del Instituto Nacional de Estadística e Informática (INEI) con la Encuesta de Hogares del 2012 (ENAHO), Tacna se encuentra en el quintil cuatro de pobreza extrema a nivel Nacional; lo cual es contradictorio ya que la Tuberculosis está relacionada a niveles de pobreza. "La pobreza no tiene una definición clara ni universal, pero está asociada a muchos aspectos de la población humana, entre ellas, las carencias, la insatisfacción de las necesidades básicas, una insuficiencia de ingresos y privación de bienes y servicios, entre otros" (INEI, 2010). El Umbral de Pobreza Extrema definido internacionalmente (Banco Mundial) como la línea fijada (2008) EN 1.25 dólares diarios per cápita (de igual poder adquisitivo) cantidad que se considera suficiente para la adquisición de productos necesarios para sobrevivir en los países de más bajos ingresos.

La estrecha relación entre malnutrición y procesos infecciosos está ampliamente reconocida y en el caso particular de la tuberculosis constituye un factor importante. En esta perspectiva el Instituto Nacional de Salud a través del Centro Nacional de

\footnotetext{
1 Magíster en Gestión y Políticas Públicas.

Medico del Centro de Salud Augusto B. Leguia.
} 
Alimentación CENAN, en coordinación con otros sectores viene ejecutando desde 1989 el Programa de Alimentación y Nutrición al paciente con tuberculosis y Familia, cuyo objetivo es contribuir a la recuperación integral del paciente ambulatorio y protección de su familia mediante el desarrollo de actividades de complementación alimentaria, educación alimentaria nutricional y evaluación nutricional, integradas a otras actividades que desarrollan los Establecimientos del Ministerio de Salud. En el Perú se diagnosticaron 25129 casos de tuberculosis en el 2012 con una incidencia de 83.4 por 100000 habitantes. Siendo el cuarto país de América con mayor incidencia de tuberculosis y el primero en severidad de la tuberculosis resistente. (INS, 2013). La Estrategia Nacional de Prevención y Control de la tuberculosis a través de su Norma Técnica asegura el tratamiento gratuito supervisado, los análisis de baciloscopía y cultivo de BK gratuitos para todas las personas afectadas de tuberculosis, en los Establecimientos de Salud del Ministerio de Salud(Ministerio de Salud, 2009). En el año 2012 según INEI con la Encuesta Nacional de Hogares (ENAHO 2012), el $6 \%$ de peruanos se encuentran en situación de pobreza extrema que equivale a 1 millón 808 mil personas con un gasto per cápita inferior al costo de la canasta básica, correspondiendo a Tacna aproximadamente 3700 personas de pobreza extrema.

El estudio realizado por Aparco y colaboradores de "Variación del estado nutricional durante el tratamiento antituberculoso en beneficiarios del PANTBC". (Aparco, Juan Pablo, Huamán-Espino, Lucio, \& Segura, Eddy R., 2012) Dice: Las mediciones se realizaron al inicio, segundo y quinto o sexto mes y se comparó la distribución del estado nutricional al inicio respecto al final (prueba de McNemar-Bowker). Se observó disminución progresiva de la condición de bajo peso $(\mathrm{IMC}<18,5)$ así como el aumento de la condición de sobrepeso. La comparación del estado nutricional al inicio respecto al final mostró cambios en la distribución $(p<0,05)$.

En el estudio de Salazar E, Figueroa C, \& Machaca T, (2005) en 2083 pacientes beneficiarios de 15 a 65 años de edad, durante julio-1996 a junio-2002 en Arequipa Metropolitana según el índice de masa corporal, los beneficiarios en general mejoraron su estado nutricional, terminando la mayoría con peso normal (de $64,53 \%$ a $84,75 \%$ ). Una proporción significativa de pacientes con tuberculosis mantiene una ingesta deficiente de energía y nutrientes a pesar de recibir alimentación complementaria. La canasta entregada no cubre el aporte de requerimientos de proteínas de alto valor biológico (Montes Jave, Cecilia \& et al, 2002). Los factores de riesgo en pacientes con tuberculosis pulmonar que fracasaron al tratamiento antituberculoso del Programa de Control de
Tuberculosis (PCT) de ocho Centros de Salud de Lima Norte durante los años 1998 y 1999 eran edad< 32 años $(O R=14,5, p=0,008)$, antecedente de contacto "de alto riesgo" $(O R=22, p<0,001)$, dísnea persistente $(O R=18, p=0,02), y$, persistencia de baciloscopía de esputo positiva al segundo mes de tratamiento $(O R=9, p=0,02)($ Meza-García \& et al, 2000).

El Programa de Alimentación y Nutrición para el paciente ambulatorio con tuberculosis y familia (PAN TBC) (ESN-PCT, 2014) viene ejecutando desde 1989 el apoyo nutricional para la recuperación integral de las personas afectadas de tuberculosis. La ración de alimentos PAN TBC cubre aproximadamente el $40 \%$ de los requerimientos calórico proteico del "Núcleo Familiar" (paciente con tuberculosis y dos contactos). Los alimentos se distribuyen mensualmente con un cálculo por ración diaria distribuida de la siguiente manera: $400 \mathrm{~g}$. de cereales corresponde $1436 \mathrm{Kcal}$. y $24.4 \mathrm{~g}$. de proteínas; $150 \mathrm{~g}$. de menestras $488 \mathrm{Kcal}$. y 30.75 de proteínas; $50 \mathrm{~g}$. de conserva de pescado $183 \mathrm{Kcal}$. con $12.8 \mathrm{~g}$. de proteínas; aceite vegetal 99 g. con $875 \mathrm{Kcal}$.

\section{OBJETIVOS}

a) Analizar el Programa de Alimentación y Nutrición para el paciente ambulatorio con tuberculosis y familia.

b) Evaluar la recuperación de personas con pobreza extrema afectadas de tuberculosis beneficiarias del Programa de Alimentación y Nutrición para el paciente con tuberculosis y familia PANTBC.

\section{METODOLOGÍA}

Investigación básica no experimental, explicativa. Se revisaron 467 Historias Clínicas de pacientes diagnosticados de Tuberculosis, encontrándose 33 personas con pobreza extrema que terminaron el tratamiento medicamentoso, con información sobre condiciones socioeconómicas, tipo de tuberculosis, evolución de la enfermedad, y a quienes se entrevistó en el último mes de tratamiento acerca del patrón de consumo de alimentos de la Canasta de víveres que reciben en forma mensual del PANTBC. Para esta investigación se utilizó la información antropométrica básica (peso, talla). La determinación del Estado Nutricional se hizo a través del Índice de Masa Corporal al inicio y final de tratamiento de los beneficiarios. 


\section{RESULTADOS}

Según el lugar de residencia, las personas con pobreza extrema en la Provincia de Tacna en el año 2013 se distribuyen de la siguiente manera: Distrito Ciudad Nueva con 26.6 casos por mil habitantes, seguido del Distrito Gregorio Albarracín Lanchipa con 14.3 casos por mil habitantes. Siendo el Distrito de Tacna el de menor número de casos con 4.5 por mil habitantes (Graf. 1).
En el graf. 2 se muestra la distribución del número de miembros del hogar. Se encuentra que en el $57.6 \%$ de las familias viven de 4 a 6 personas y en el $33.3 \%$ de 1 a 3 personas.. En el grupo de pobreza extrema afectadas de tuberculosis, consumen lentejas de 3 a 4 veces por semana el $51.5 \%$, y de 5 a 6 veces por semana $30.3 \%$. Consumen pescado en conserva 1 a 2 veces por semana $54.5 \%$ y de 3 a 4 veces por semana el $27.3 \%$ (Graf. 3 ). El $60.6 \%$ consume arroz 5 a 6 veces por semana (Graf 4).

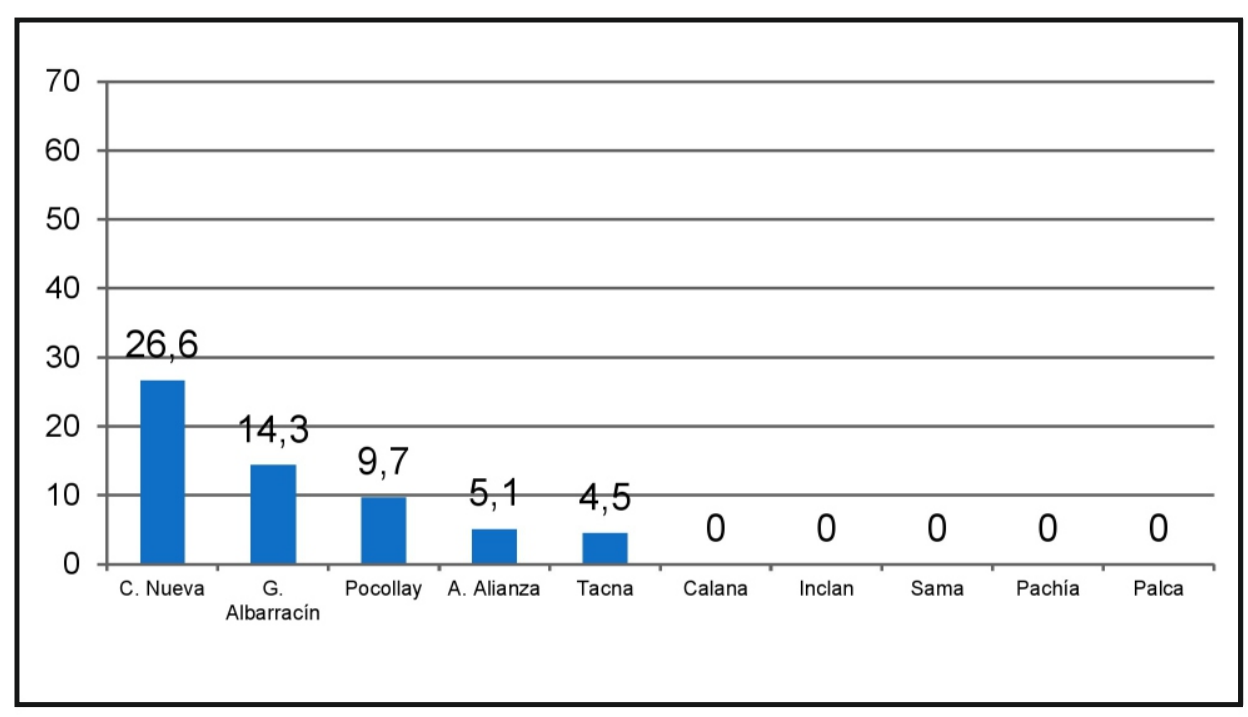

Graf. 01. Distribución de personas con pobreza extrema

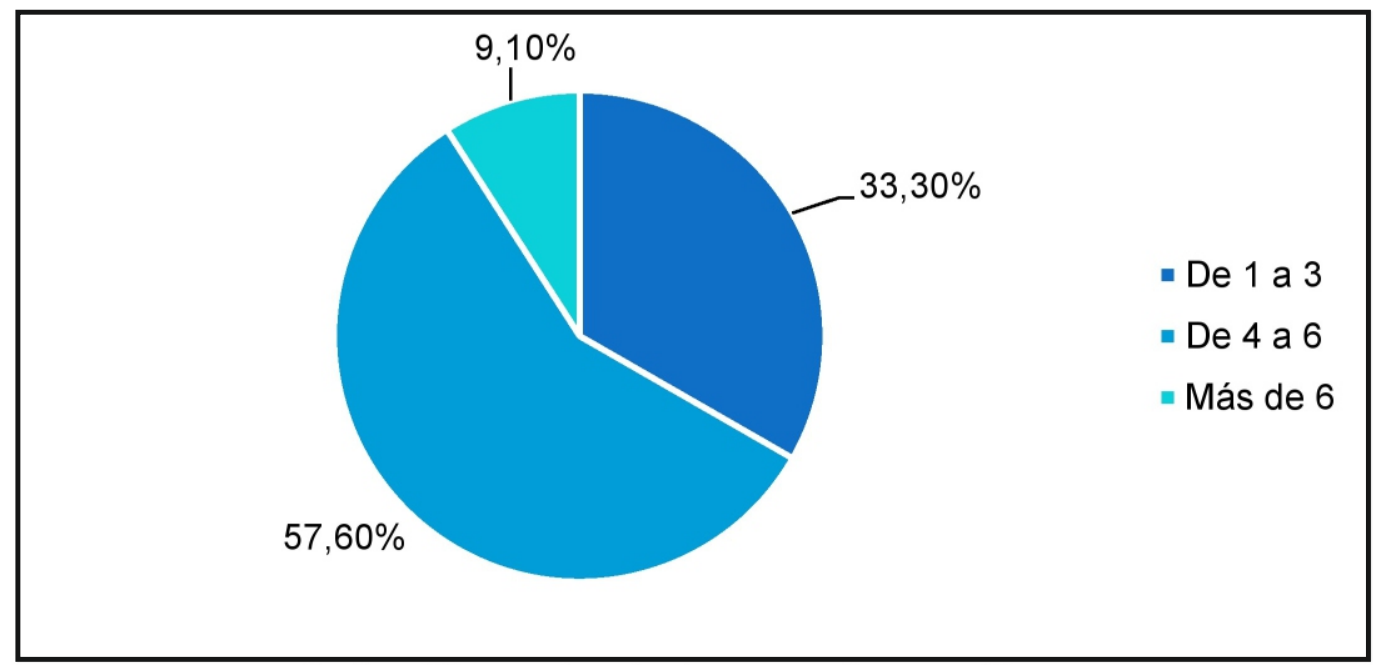

Graf. 2: Número de miembros que viven en el hogar 


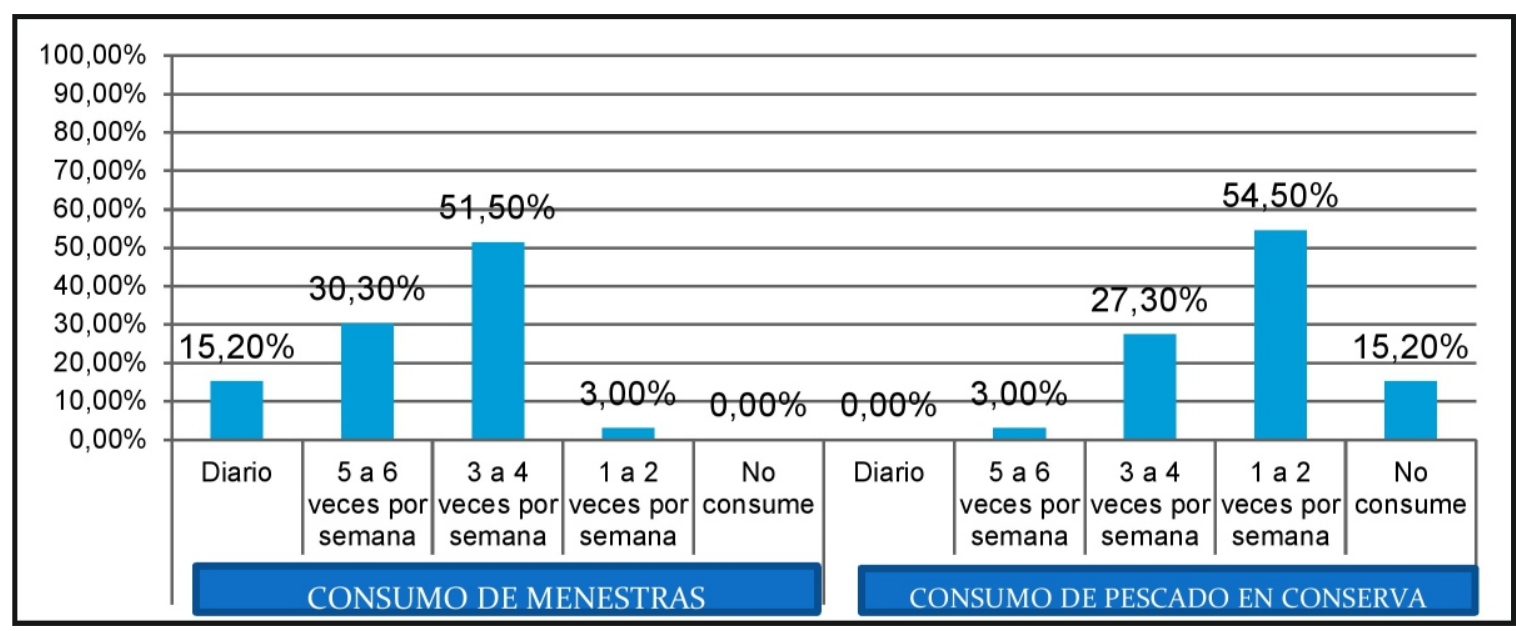

Graf. 3: distribución de la frecuencia del consumo de menestras y pescado

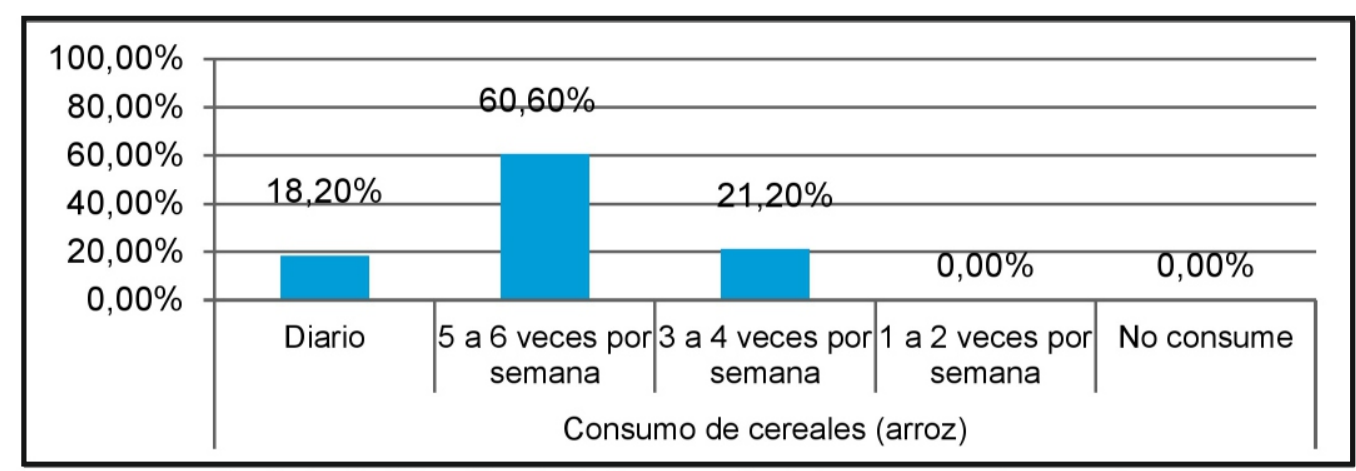

Graf. 4: distribución de la frecuencia del consumo de cereales

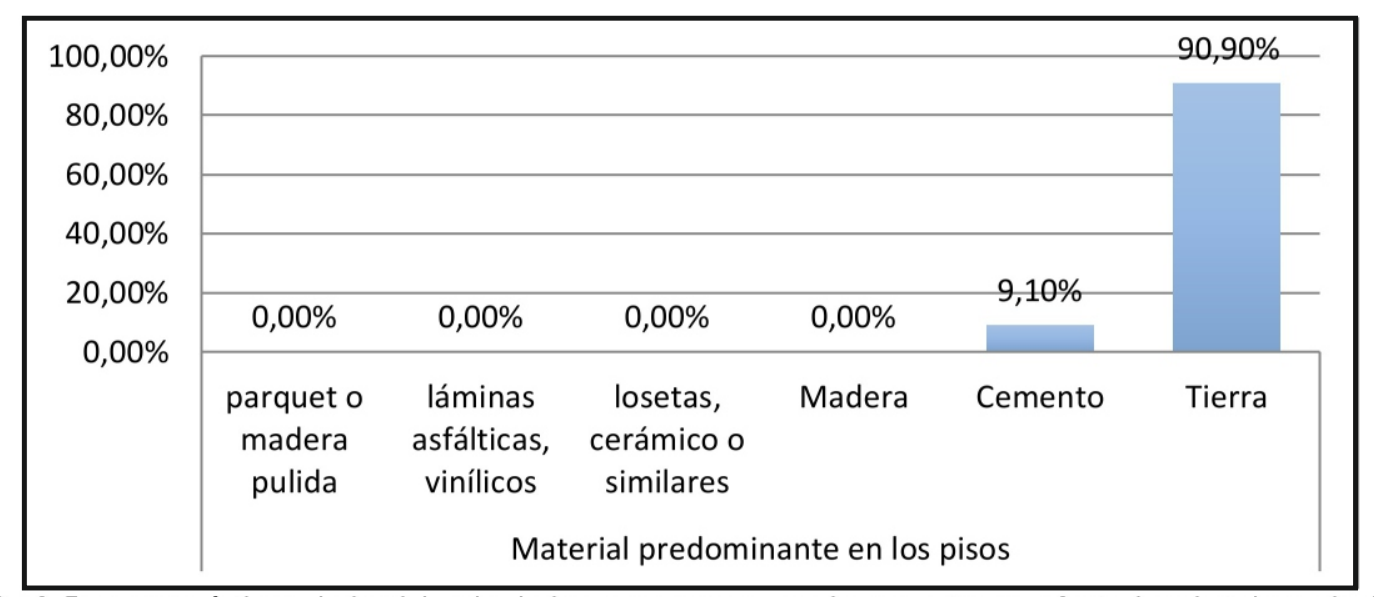

Graf. 5: características de la vivienda de las personas con pobreza extrema afectadas de tuberculosis.

La Graf. 5. muestra las características de la vivienda. El material predominante en los pisos es la tierra $(90.9 \%)$ A nivel nacional según, el INEI publica que, en los pobres extremos, el $86.9 \%$ de viviendas son con piso de tierra.
En esta figura se muestra el IMC al inicio y final del tratamiento. la mayoría de personas tiene índice de masa normal al término del tratamiento con el $60.6 \%$. En este gráfico se observa la variación del índice de masa corporal de personas al inicio del tratamiento con respecto al término, encontrando que las 
personas con bajo peso variaron del $30.3 \%$ al inicio del tratamiento al $9.1 \%$ al término. Aquello con peso normal continuó en esa misma condición al finalizar el tratamiento. El $6.10 \%$ del grupo en estudio ingresó a tratamiento con diagnóstico de sobrepeso, aumentando esta proporción a $30.3 \%$ al finalizar el manejo.

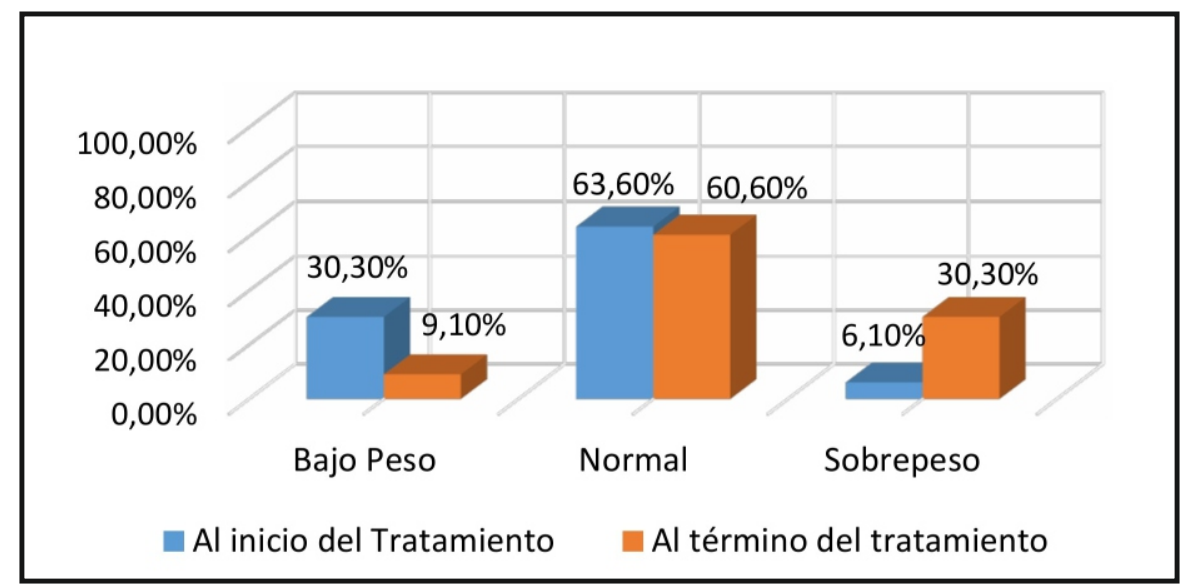

Graf. 6: Indice de masa corporal al inicio y término del tratamiento en personas con pobreza extrema afectadas de tuberculosis

\section{DISCUSIÓN}

En la Provincia de Tacna en el año 2013 la Estrategia Nacional de Prevención y Control de Tuberculosis (ESNPCT) (MINSA, 2013), reportó 467 casos nuevos de tuberculosis. Del total de estos casos, 33 personas eran de pobreza extrema y estaban afectadas de tuberculosis. La mayoría de personas en esta condición pertenecían al distrito de Ciudad Nueva, con una tasa de 26.6 casos por mil habitantes, seguido del Distrito de Gregorio Albarracín con una tasa de 14.3 casos por mil habitantes. El hacinamiento estuvo presente en el $33.3 \%$. En el estudio de Quispe López, Jorge Luis., (2010) se encontró hacinamiento en el $37 \%$ en el año 2009 en los pacientes tratados en Tacna.

El $87.9 \%$ de las personas reciben sus alimentos en el desayuno, almuerzo y cena en sus propias casas. El consumo de alimentos de la canasta PANTBC en forma diaria es bajo, alcanzando el $15.2 \%$ en el consumo de menestras, pescado en conserva en forma diaria es $0.0 \%$, cereales el $18.2 \%$ y aceite el $97 \%$, lo que significa que el incremento de peso podría explicarse por el aumento de apetito e ingesta de alimentos, la reducción de las demandas de energía y la mejora de la eficiencia metabólica, entre otras razones. Existe escasa investigación sobre la evaluación del PANTBC en los beneficiarios durante su tratamiento.

La evaluación del impacto del Programa PANTBC realizada por (Asociación Benéfica PRISMA., 2002) en el 2010 encontró reducción de la proporción de beneficiarios con bajo peso, poca variación en la categoría normal y aumento en sobrepeso, sin embargo, no se consideró en el análisis la condición nutricional inicial en los beneficiarios. En el estudio de Salazar E et al., (2005) se evaluó la variación del estado nutricional en beneficiarios del PANTBC en Arequipa en forma retrospectiva a partir de registros de 1996 al 2002 y encontró que a lo largo del tratamiento el porcentaje de personas con bajo peso disminuyó de $25 \%$ al $14 \%$, mientras que el peso normal aumentó de 64 a $84 \%$, en tanto que el sobrepeso se redujo en $100 \%$.

La Región Tacna se encuentra en el Quintil Cuarto de pobreza extrema a nivel nacional, según la Encuesta Nacional de Hogares 2012 (ENAHO) (INEI, 2015); sin embargo, la tuberculosis sigue ocupando el cuarto lugar después de Lima, Callao, Madre de Dios. Aquí se evidencia una contradicción ya que la tuberculosis siempre se relaciona a niveles de pobreza y número de miembros en la familia.

Actualmente todas las personas con diagnóstico de tuberculosis son elegibles para recibir la canasta alimentaria independientemente de su estado nutricional y composición familiar al momento del ingreso al Programa. EI PANTBC no considera el incremento de la canasta de víveres para las familias de pobreza extrema, a pesar de contar con información suficiente de las condiciones socio económicas, tener el reporte mensual de los casos nuevos.

En el $90.9 \%$ el peso aumenta, permanece igual en el $9.1 \%$, en ninguno disminuye el peso. El indicador del PANTBC es la proporción de pacientes recuperados, es decir el número de pacientes con bajo peso al inicio 
del tratamiento menos el número de pacientes con bajo peso al término de tratamiento entre el total de pacientes de bajo peso que tienen controles por 100 , sin tomar en cuenta los abandonos al tratamiento. El $70 \%$ de pacientes con bajo peso se recuperan, aplicando el indicador del PANTBC.

El Índice de Masa Corporal sólo determina el peso ideal para la estatura, por tanto, no es suficiente para evaluar realmente la recuperación nutricional en estos pacientes. Según el índice de masa corporal la mayoría de beneficiarios mejoraron su estado nutricional. Sin embargo, según el número de miembros en los hogares en estudio encontrado es de 4 a más personas por lo que se puede deducir que el apoyo alimentario del PAN TBC en estos pacientes no cubren el aporte calórico proteico esperado, ya que en $66.7 \%$ de familias están integradas por 4 a más personas y el objetivo del PAN TBC es aportar aproximadamente el $40 \%$ del requerimiento calórico proteico teniendo en cuenta como núcleo familiar beneficiario sólo a 3 personas. Es precisamente en estas personas afectadas de tuberculosis con pobreza extrema que más requieren el aporte nutricional que no lo reciben en forma completa por el PAN TBC.

Es importante mencionar que este estudio permite conocer los patrones de consumo de los alimentos del PANTBC por los beneficiarios, encontrando que la canasta de víveres de este programa no cubre el requerimiento calórico proteico esperado, que es aproximadamente del $40 \%$; debido principalmente a la dilución de las raciones de alimentos, ya que se encuentra un mayor porcentaje de personas con pobreza extrema afectadas con tuberculosis que viven en hogares de 3 a más miembros.

No se encuentran estudios relacionados a la dilución de las raciones de la canasta del programa PANTBC para realizar comparaciones con respecto a los beneficiarios en general. Los resultados del estudio muestran que existe un cambio del Estado Nutricional y un aumento significativo del Índice de Masa Corporal en los beneficiarios de Programa de Alimentación y Nutrición para paciente ambulatorio con Tuberculosis y familia en personas con pobreza extrema.

\section{BIBLIOGRAFÍA:}

Aparco, Juan Pablo, Huamán-Espino, Lucio, \& Segura, Eddy R. (2012). Juan Pablo Aparco, Lucio Huamán-Espino, Eddy R. Segura. Estudio de Variación del estado nutricional durante el tratamiento antituberculoso en beneficiarios del programa PANTBC, Revista Peruana de Medicina Experimental y Salud Publica Vol 29 (3).
Asociación Benéfica PRISMA. (2002). Asociación Benéfica PRISMA. Informe Final: Evaluación del Impacto Nutricional para el PANTBC 2002, Lima: Asociación Benéfica Prisma 2002.

ESN-PCT. (2014). Documento técnico. formulacion de la ración alimentaria del programa de complementacion alimentaria para la personba afectada por tuberculosis. Ministerio de Salud. Resolucion ministerial 653-2014. MINSA. Recuperado 18 de junio de 2017, a partir de ftp://ftp2.minsa.gob.pe/normaslegales/2014/RM653_ 2014_MINSA_b.pdf

INEl. (2010). Metodología para la medición de lapobreza en el Perú. Metodologías estadísticas. Recuperado 18 de junio de 2017, a partir de https://www.inei.gob.pe/media/MenuRecursivo/metod ologias/pobreza01.pdf

INEI. (2015). Mapa de pobreza provincial y distrital 2013. Instituto Nacional de estadística e informática. Setiembre. Encuesta Nacional ENAHO. Recuperado a partir de https://www.inei.gob.pe/media/MenuRecursivo/public aciones_digitales/Est/Lib1261/Libro.pdf

INS. (2013). nstituto Nacional De Salud. Boletin Epidemiologico 2013 Vol 6 Núm 3.

Meza-García, M., \& et al. (2000). Factores de riesgo para el fracaso del tratamiento antituberculoso totalmente supervisado.

Ministerio de Salud. (2009). Plan Estratégico Multisectorial 2010 - 2019 Para Detener La Tuberculosis En Perú.

MINSA. (2013). Norma técnica de salud para el control de la tuberculosis / Ministerio de Salud. Dirección General de Salud de las Personas. Estrategia Sanitaria Nacional de Prevención y Control de la Tuberculosis -- Lima: Ministerio de Salud; 2013 p.; ilus. Recuperado a partir de ftp://ftp2.minsa.gob.pe/descargas/dgsp/ESNtuberculosis/normaspublicaciones/NTSTBC.pdf Montes Jave, Cecilia, \& et al. (2002). Patrones de consumo de alimentos del programa PANTBC.

Quispe López, Jorge Luis. (2010). factores asoicados al fracaso del tratamiento antituberculosos en los pacientes de la provincia de Tacna, tratados durante los años 2006 - 2009. (Tesis). Universidad Nacional Jorge Basadre Grohmann de Tacna, Tacna. Recuperado a partir de http://redi.unjbg.edu.pe/bitstream/handle/UNJBG/476 /TG0333. pdf? sequence=1\&isAllowed=y

Salazar E, V., Figueroa C, R., \& Machaca T, R. (2005). Variación del estado nutricional de los beneficiarios del programa pantbc en Arequipa metropolitana, 1996 - 2002. Revista Peruana de Medicina Experimental y Salud Publica, 22(2), 134-138.

Recibidos: 09 / 05 / 17

Aceptado para publicación: 09 / 06 / 17 